\title{
Ultrastructural organization of the tomato embryo sac at the time of fertilization
}

\author{
FRANCISZEK KADEJ, AGNIESZKA KADEJ \\ Central Laboratory of the Maria Curie-Sklodowska University, \\ ul. Akademicka 19, 20-033 Lublin, Poland
}

\begin{abstract}
In tomato embryo sac at the time of fertilization noticeable multiplication of the endosperm cytoplasm is observed. The cytoplasm of the egg cell, next to the nucleus, appears loose with distinct mitochondria and plastids. At a distance from the nucleus ribosomes become grouped into a cytoplasmic mass of great uniformity. Nucleolar structure of the egg cell nucleus displaces to its periphery. Its new position seems to be related to the process of fertilization. In the area of the endosperm two nuclei in close contact to each other are found which suggests the beginning of secondary fertilization.
\end{abstract}

\section{INTRODUCTION}

Investigations on the fertilization process in plants at the electron microscope level take into account mainly stages before or/and after fertilization (among others Schulz, Jensen, 1968; C ocucci, Jense n, 1969; Mog ens en, 1972, 1978; Linskens, 1974; B ell, 1975). In view of technical difficulties, every stage close to the fertilization process should be given careful attention.

\section{MATERIAL AND METHODS}

Ovules of Lycopersicon esculentum (Miller) were fixed 44 hours after pollination in $4 \%$ glutaraldehyde buffered with $0.06 \mathrm{M}$ phosphate at $\mathrm{pH} 7$, for $2 \mathrm{hr}$ at $0^{\circ} \mathrm{C}$, post-fixed in $2^{\%}$ unbuffered $\mathrm{OsO}_{4}$ for $2 \mathrm{hr}$ at $0^{\circ} \mathrm{C}$ and embedded in Araldite. Ultrathin sections were examined with Tesla EM 613.

\section{OBSERVATIONS}

Forty four hours after pollination, the endosperm shows a considerable increase of cytoplasm and contains more than one nucleus (Fig. 9). 
Figs 1, 2. Portion of cytoplasm of the endosperm cell. In the plastids starch autolysis is seen (arrows)

er - endoplasmic reticulum; lp — lipid body; pl - plastid; v - vacuole; $\mathrm{m}$ mitochondrion. $\times 30000$

Figs 3, 4. The egg apparatus of the same embryo sac at two different depths The single arrow indicates the persistent synergid. The degenerating synergid is filled with pollen tube material (pt). Double arrows show the localization of a uniformly granular ribosomal area. Fig. $3 \times 4500$, Fig. $4 \times 6000$.

Fig. 5. Portion of egg cell, the double arrow lies in the ribosomal area. $\times 10000$. Fig. 6. Portion of egg cell which probable presents a zygote

\section{d - dictyosome. $\times 1000$.}

Fig. 7. Micropylar portion of the embryo sac

Circles show the sites of the egg cell nucleus (in the middle) and two endosperm nuclei visible in the neighbouring sections of the same series. The darker body in the pollen tube material is considered as one of the sperm nuclei (arrow). $\times 5000$.

Fig. 8. Portion of egg cell

nu - deformed egg cell nucleus; probably after fertilization with peripheral nucleolar structure. Note bright inclusion in close contact to them (arrow). $\times 8000$.

The dictyosomes ,mitochondria and endoplasmic reticulum (ER) keep their typical ultrastructure and are rather scarce in number. In some plastids, symptoms of starch autolysis can be seen (Figs 1, 2).

Whithin the egg apparatus one of the synergids has been completely filled with pollen tube material (Fig. 3). The other, persistent synergid, is rich in ribosomes, long cisternae of ER (Fig. 4) and other organelles.

The egg cell, most likely zygote by now, is covered by a thin, loose cell wall (Figs 3, 4, 7, 8). Occasionally some bodies persist being probable the remains of the filiform apparatus (Fig. 10). The central vacuole of the egg cell disappears and is replaced by a uniformly granular and relatively dense cytoplasmic mass (Figs 3,4) containing ribosomes only (Fig. 5). In that area a well-developed system of ER, commonly arranged in parallel cisternae, resembling ergastoplasm, is often formed (Fig. 6). In their closest vicinity numerous small vesicles, dictyosomes and mitochondria are assembled.

In the period of fertilization (and somewhat prior to it) a part next to the nucleus of the egg cell is occupied by a highly loose cytoplasm with small vacuoles, plastids and mitochondria (Figs 4, 5). Within the nucleus no nucleolus is found in the central position. However, the nucleus is characterized by an osmophilic dense structure on its periphery (Figs 4, 5). Successive sections indicate that the localization of the osmophilic structure seems to be related to the structure considered as a trace of pollen tube material (Figs 10, 8).

At the same developmental stage, a body closely connected with one of the endosperm nuclei is observed (Figs 10, 11). We assume it to be 

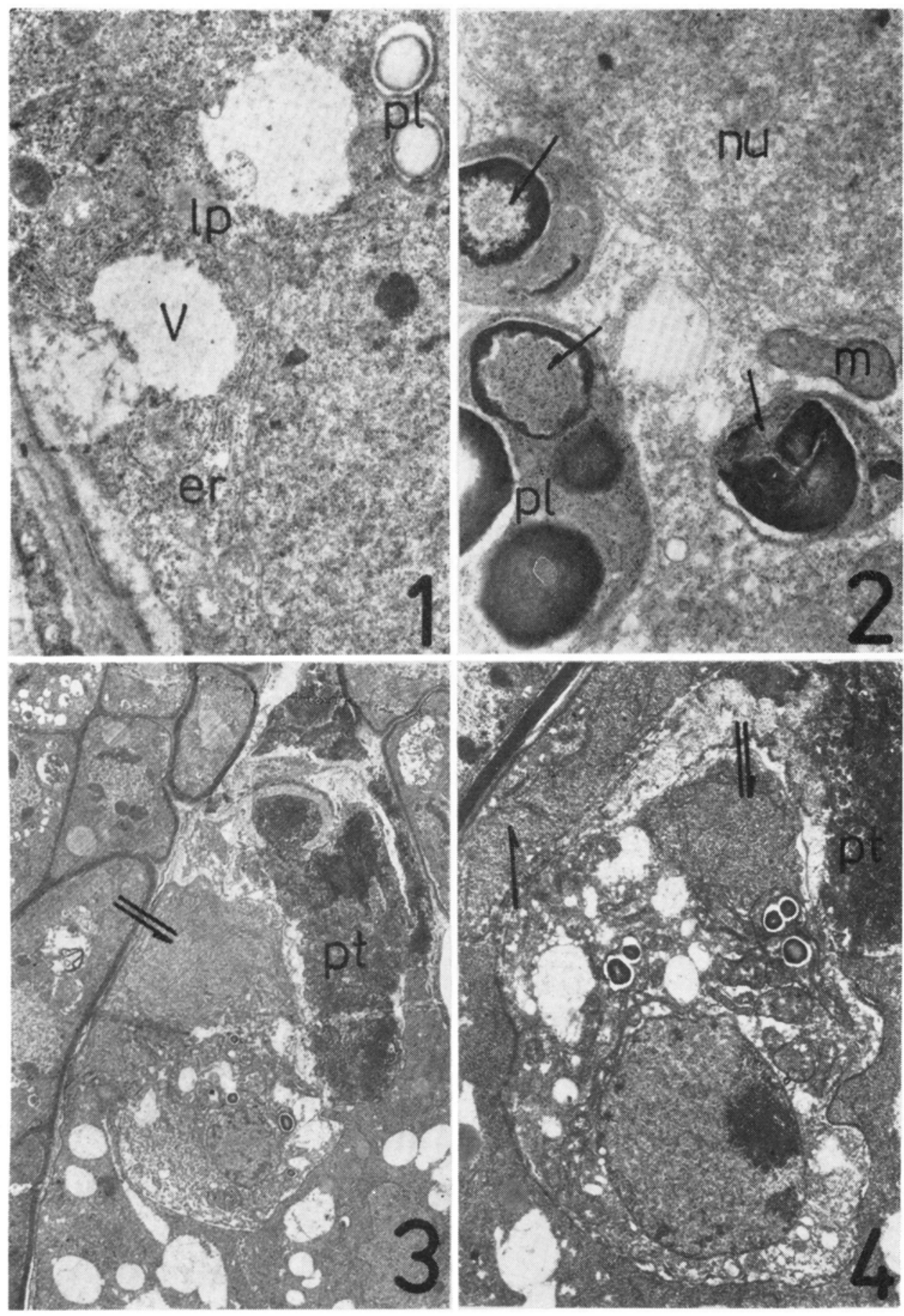


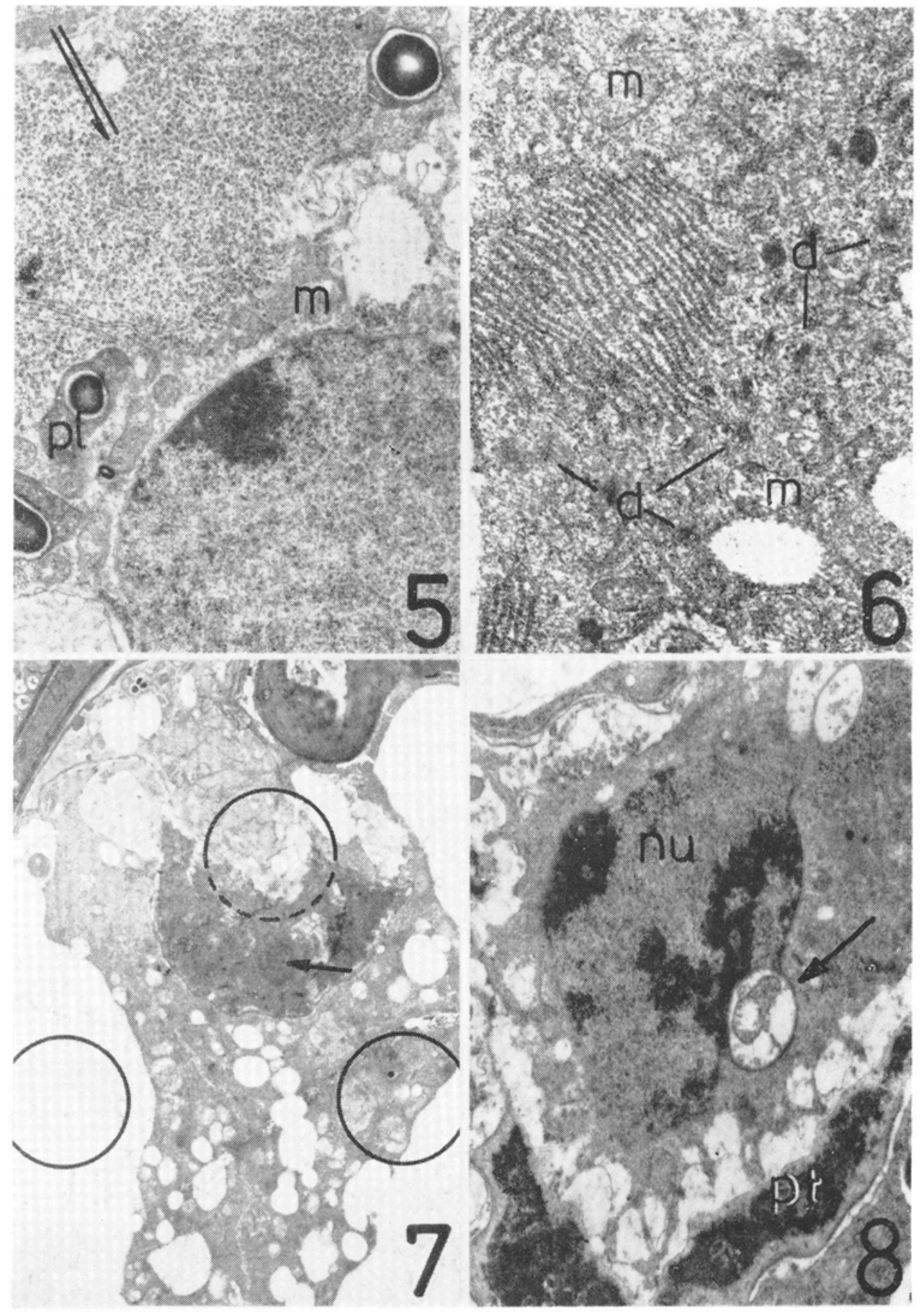




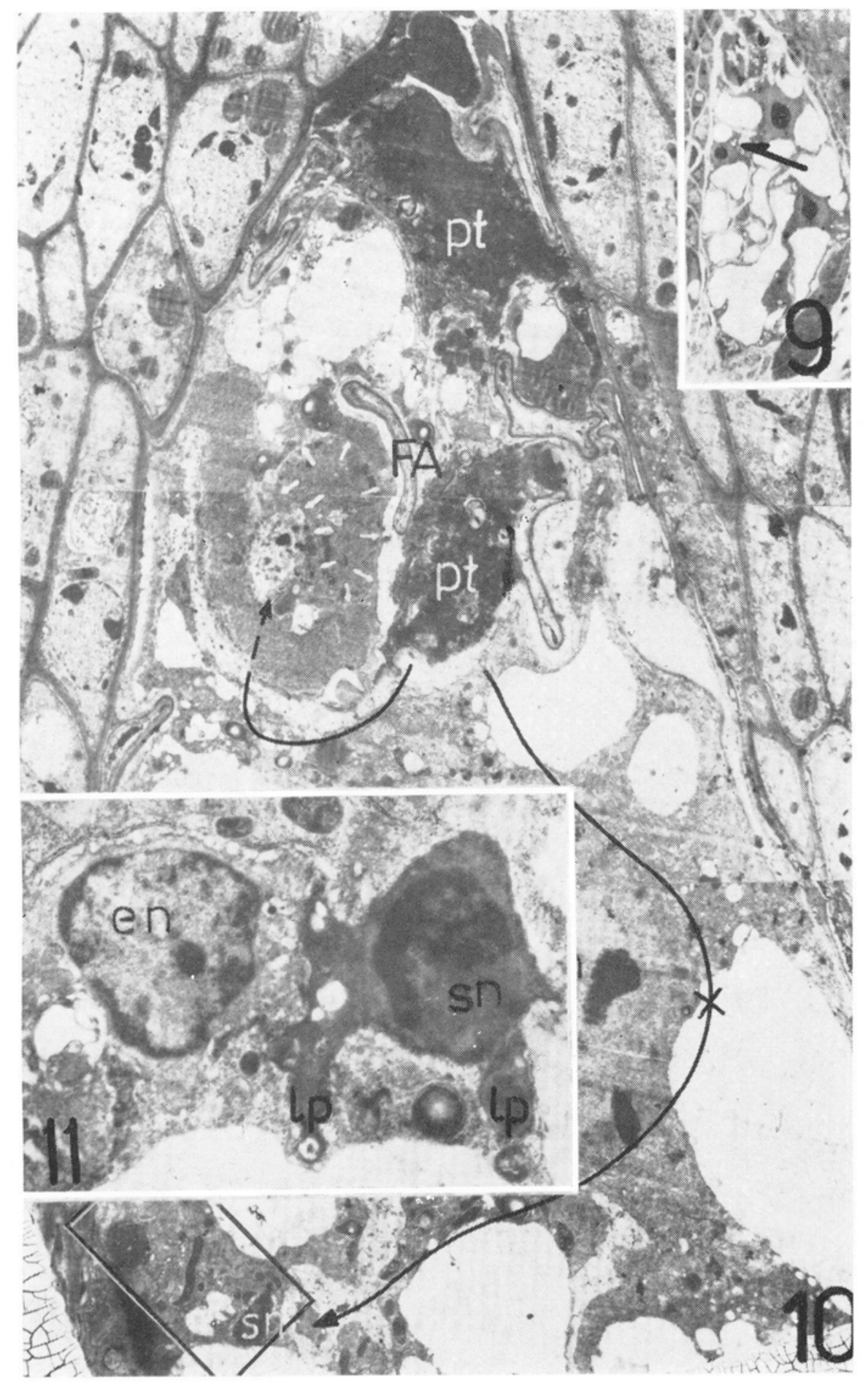


Fig. 9. Embryo sac in semithin section. At its top the egg apparatus The arrow shows the site of the sperm nucleus visible in next sections of the same series. $X$ ca 200 .

Fig. 10. Next ultrathin sections of the same series shown in Fig. 9

pt - material of the same pollen tube. Arrows show the supposed course of the sperm nuclei. The membrane of the egg cell (zygote) nucleus is shown by white arows. In the rectangle the remains of the sperm nucleus. $\times 3600$ Fig. 11. The same sperm nucleus $(\mathrm{sn})$ as shown in Fig. 10 medially sectioned in contact to the endosperm nucleus (en). $\times 8000$

a sperm nucleus at the beginning of secondary fertilization. The nucleus is filled with relatively dense material in which a darker centre can be distinguished. On the periphery of this sperm nucleus tiny vacoules and lipids can be seen (Fig. 11).

\section{DISCUSSION}

The ultrastructure of the tomato embryo sac at the time of fertilization is generally similar to that of megagametophyte of Epidendrum following fertilization described by Cocucci and Jensen (1969). A great resemblance is found particularly in the fine structure of the egg cell almost at the same development stage. As in Epidendrum, organelles in the young tomato zygote are marked and their membranes are more distinct. However, in contrast to Epidendrum, ER and dictyosomes might have disappeared before fertilization because they are absent in many egg cells at a certain stage of the embryo sac development. The ribosomal system may also undergo a considerable dissolution or displacement. The new ribosomal system forms uniform large areas, usually at a distance from the nucleus (Figs 3, 4, 5). In these areas full of ribosomes, ER cisternae and resembling ergastoplasm are formed (Fig. 6). It is interesting that large numbers of dictyosomes and mitochondria are grouped around the ergastoplasm. The above changes seem to account for a deep functional reorganization in the young zygote.

The fertilization of the egg cell must have occurred a very short time ago which is evidenced by considerable deformation of the nucleus shape of the young zygote, dislocation of the nucleolar structure on its periphery (Figs 4, 5, 6), as well as by the structure shown in Fig. 8, absent in any other nuclei. A question arises whether the dislocation of the nucleolus on the periphery is related to the sperm nucleus in the fertilization process.

Lycopersicon belongs to the group of plants in which the endosperm can develops apart from secondary fertilization process (S o ln tzeva, 1978). The fact that the development of the endosperm occurs apart 
from secondary fertilization makes its identification very difficult. If the body in the area of the endosperm is the sperm nucleus, its structure, different from that of the endosperm nuclei will advance further studies on fertilization process.

\section{REFERENCES}

Bel1 P. R. R., 1975. Observations on the nucleus during fertilization. J. Cel: Sci. 17: 141-153.

Cocucci A., Jensen W. A., 1969. Orchid embryology: megagametophyte of Epidendrum scutella following fertilization. Amer. J. Bot. 56: 629-640.

Linskens H. F., 1974. Fertilization in Higher Plants In: Proceedings of the International Symposium on Fertilization in Higher Plants. North-Holland Publishing Company, Amsterdam.

Mogensen H. L., 1972. Fine structure and composition of the egg apparatus before and after fertilization in Quercus Gambelli: the functional ovule. Amer. J. Bot. 59: 931-941.

M o gensen H. L., 1978. Pollen tube-synergid interaction in Proboscidea louisianica (Martneaceae). Amer. J. Bot. 65: 953-964.

$\mathrm{Schulz}$ P., Jensen W. A., 1968. Capsella embryogenesis: the synergids before and after fertilization. Amer. J. Bot. 55: 541-552.

Solntzeva M. P., 1978. Apomixis and hemigamy as one of its forms. Proc. Indian Natur. Sci. Acad. B 44: 78-90. 